\title{
Study on the Photosynthetic Efficiency of the Dracontomelon Duperreanum Leaves by Using Photo-acoustic Tomography Spectroscopy
}

\author{
Gan-Wen LIE ${ }^{1, a}$, Guang-Hua LIE ${ }^{2, b,{ }^{*}}$, Song-Xi LIU ${ }^{2, \mathrm{c}}$, Wen-Chang DENG ${ }^{2, \mathrm{~d}}$, \\ Jian-Chao LIANG ${ }^{3, e}$
}

${ }^{1}$ College of Zhujiang, South China Agricultural University, Guangzhou 510642, People's Republic of China

${ }^{2}$ College of Physical Science and Technology, Lingnan Normal University, Zhanjiang 524048, People's Republic of China

${ }^{3}$ Guangdong Entomological Institute, Guangzhou 510260, People's Republic of China

acCartonLie@163.com, biegh88@163.com, '969797029@qq.com, d836051670@qq.com,

e13760813102@163.com

${ }^{\star}$ Corresponding author

Keywords: Dracontomelon duperreanum, Photo-acoustic tomography spectroscopy, Green leaves, Yellow leaves, Photosynthetic efficiency.

\begin{abstract}
By using a new kind of single-beam normalized photo-acoustic tomography spectroscopy (PAS-CT) technology with non-damage detection, the photo-acoustic tomography spectrum, optical absorption properties and photosynthetic pigment content of the green and yellow leaves of Dracontomelon duperreanum were studied. The photo-acoustic tomography spectrograms, obtained from different chopping frequency and sample translocation, were perfectly conform to each layer of cell's optical absorption properties in Dracontomelon duperreanum leaves. The results showed that: the more photosynthetic pigment content the leaves of Dracontomelon duperreanum contain, the bigger optical absorption coefficient and the higher photosynthetic efficiency they have. In the research, we could find that the photosynthetic pigment content of the green leaves of Dracontomelon duperreanum is higher than that of the yellow ones. As a result, the optical absorption coefficient and the photosynthetic efficiency of the green ones were higher than the yellow ones. The photo-acoustic tomography spectroscopy could be a kind of non-damage detection to confirm the growth of trees. According to the results of our research, the photosynthetic efficiency of Dracontomelon duperreanum could be improved and its growth time could be effectively controlled. And it provides foundations to cultivate Dracontomelon duperreanum effectively. Furthermore, it could make benefits to the controlled environmental forestry and increase forest production to meet the need of forest for 7 billion people. The research showed high science value to the study and application of the photosynthesis of plants.
\end{abstract}

\section{Introduction}

Photo-acoustic tomography spectroscopy (PAS-CT) is a new kind of spectroscopic technique with non-damage detection. If we measure the spectral absorption coefficient of leaves by the traditional absorption spectrometry, obstacles would be met by the interference of scattered light. As the photo-acoustic spectroscopy measures the heat from the material after absorption of light which is irrelevant to the scattered light, its influence could be avoided. So it is suitable for the spectral detection and analysis of strong scatter and opaque substances such as leaves, particles, powders, stains and other strong scattering turbid liquid. this methods can avoid. It will be a new method to study photosynthetic efficiency of plant. And it has the advantages of high sensitivity, high universality and non-destructive measurement. As a result, it will be widely applied in material science [1-3], biomedical [4-8], agriculture and forestry and other fields. In this paper, the 
photo-acoustic tomography spectroscopy (PAS-CT) of Dracontomelon duperreanum leaves and its chromatography optical absorption coefficient were measured. The study of the optical properties of leaves is to reveal a more effective way to detect the growth of trees in non-damaging way, and improve the leaves’ capacity of absorbing light.

\section{Experimental Principle of Photoacoustic Tomography Spectroscopy}

According to Rosencwaig-Gersho (R-G) theory [9,10], when the substances (samples) modulators are alternating light heating, some of the heat flow through the thermal conductivity of gas with it. As the attenuation of the heat wave is a fast wave, only the thin surface layer of material in contact with the gas get this kind of alternating heat. And by heat, this thin layer, as the alternating heating and vibration undergo, like a vibration pistons firing sound waves, generate the photo-acoustic signal. $\mathrm{R}-\mathrm{G}$ is based on the theory. Suppose the sample and reference samples testing photo-acoustic signals are $S_{s}(\lambda), S_{c}(\lambda)$ :

$$
\begin{aligned}
& S_{s}(\lambda)=C I(\lambda) \beta_{s}(\lambda) \\
& S_{c}(\lambda)=C I(\lambda) \beta_{c}
\end{aligned}
$$

Samples which are used and the reference sample absorption spectrum coefficients is $\beta_{s}(\lambda), \beta_{c}$. $C$ is the ratio of the experimental conditions of the coefficients. $I(\lambda)$ is the light intensity. As the reference sample (carbon black) of the absorption coefficients $\beta_{c}$ is not related to the wavelength, which $\beta_{c}$ is a constant [11], and so the sample of carbon black by the photo-acoustic signal intensity only with the light source, and the wavelength. Photo-acoustic signal of carbon black $S_{c}(\lambda)$ is the power spectrum of xenon lamp. In order to eliminate the power spectrum of the light source, we use the normalized photo-acoustic spectroscopy, which uses photo-acoustic signal of the sample divided by the photo-acoustic signal of carbon black:

$$
\frac{S_{s}(\lambda)}{S_{c}(\lambda)}=\frac{\beta_{s}(\lambda)}{\beta_{c}}
$$

The sample normalized photo-acoustic spectroscopy can be obtained.

In biology, photosynthesis of green plants and some bacteria play a important role in photochemical. Normalized photo-acoustic signal $q$ can be written as[12,13]

$$
q=a\left[1-\sum_{i}\left(\frac{\eta_{i}^{\prime} \Delta E_{i}}{N h v}\right)-\left(\frac{\eta v_{f}}{v_{e}}\right)\right]
$$

Here $a$ is the part of absorbed light energy of samples. $a$ was expressed as $\frac{S_{s}(\lambda)}{S_{c}(\lambda)}=\frac{\beta_{s}(\lambda)}{\beta_{c}} .^{\prime}{ }^{\prime}$ is the quantum yield of photochemical reaction, this reaction $\Delta E_{i}$ is the formation of each product can change within the mole, $\eta$ is the quantum yield of luminescence, $v_{f}$ and $v_{e}$ all the radiation and absorption of light frequencies, $N$ is Avogadro constant, but $h$ is the Planck constant.

As is known to all. Organisms capture solar energy and transform it into chemical energy of reaction is called photosynthesis, its most general form 


$$
\mathrm{H}_{2} \mathrm{D}+\mathrm{A}-\stackrel{\text { Light }}{\rightarrow} \mathrm{H}_{2} \mathrm{~A}+\mathrm{D}
$$

Here $D$ is the electron donor, $A$ is the electron acceptor. Photosynthesis occurs only in green plants, algae, certain protists and bacteria cells that contain chlorophyll. The photosynthesis of plants are proceed in the chloroplast. And chlorophyll is the primary pigment for plants' photosynthesis, which has the similar structure with protoheme. Energy is absorbed by chlorophyll from ligh, and used to turn carbon dioxide and water into carbohydrates.

As a result, when it comes to absorption spectrum, there are Soret band near 420nm, carotenoid band between $450 \mathrm{~nm}$ and $550 \mathrm{~nm}$, and chlorophyll band between $600 \mathrm{~nm}$ and $700 \mathrm{~nm}$ in the visible range can integrallty expressed the optical properties of photosynthetic pigments. By using photo-acoustic tomography spectroscopy, the photo-acoustic absorption spectrum of photosynthetic pigments could be obtained from ordinary leaves without separation.

\section{Biological Materials and Test Methods}

\section{Biological Materials}

Fresh and clean green leaves and yellow leaves from upper main branch of Dracontomelon duperreanum were picked. According to the measurement requirements, small pieces of green and yellow leaves of Dracontomelon duperreanum near the main vein on the left side were cut into the size of the sample chamber of the photo-acoustic cell, and were measured immediately. The whole process was less than 20 minutes.

\section{Test Method of Biology Material}

Photo-acoustic tomography spectroscopy is to detect photo-acoustic signals in different layers by changing the chopper frequency and sample translocation. And then their optical absorption properties are obtained.

\section{Experimental Methods and Device}

\section{Experimental Device}

Different from the commercially available double-beam photo-acoustic spectrometer, we used single-beam normalized photo-acoustic tomography spectroscopy experimental setup (as shown in Fig.1). The light source is $1000 \mathrm{~W}$ xenon lamp, mono-chromator, Beijing Optical Instrument Co., Ltd. Zhuo-li Han's Omni- $\lambda 300$ line grating spectrometer, lock-in amplifier Stanford Research System for U.S. companies SR530 lock-in amplifier type. Data acquisition system for the Beijing Optical Instrument Co., Ltd. DCS103 Zhuo-li Han's data acquisition system. Photo-acoustic cell is developed by the gas microphone photo-acoustic sensor.

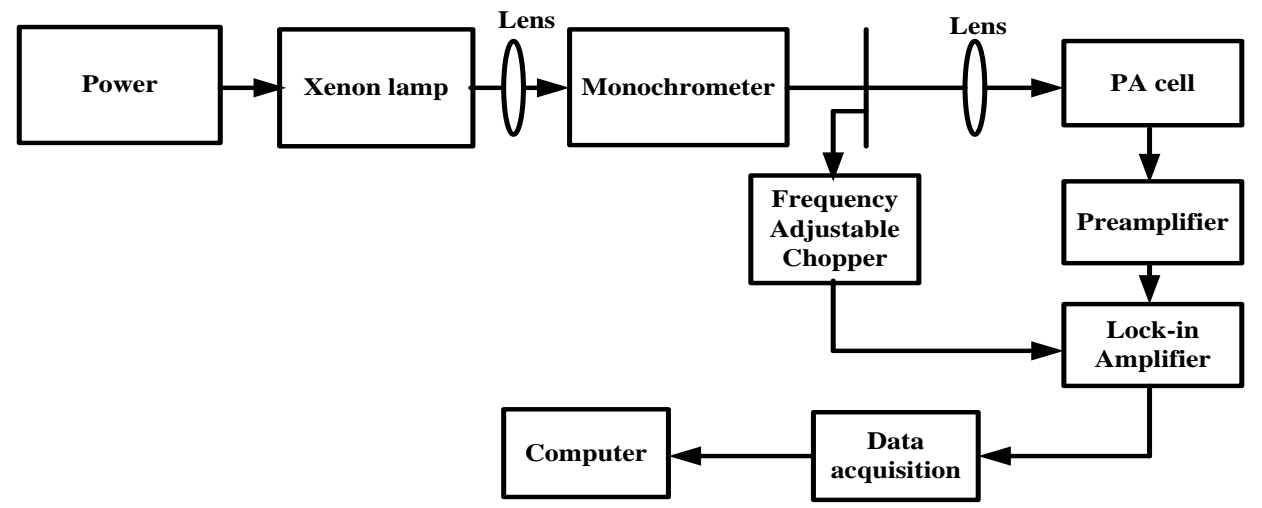

Fig.1 The experimental setup of single-beam normalized photo-acoustic tomography spectroscopy (Chopper Frequency Adjustable and Samples Were Displaced) 
White light is given by the monochromator, after splitting into the monochromatic light. Modulated by the chopper into a cyclical change in intensity modulated light, modulated light focused by the lens to the Photo-sound-pool (PA cell). Modulated light in PA cell generates photo-acoustic signal amplified by the preamplifier to the lock-in amplifier to amplify transmission and processing. The lock-in amplifier output signal is collected and stored by the computer.

\section{Measuring Method}

In order to obtain samples of the normalized photo-acoustic tomography spectroscopy, first take carbon black as a test sample, because the absorption of carbon black is not related to the wavelength (ie the absorption coefficient is a constant).Thus the xenon lamp spectrum is obtained. Then, in the same experimental conditions (voltage, operating current, chopping frequency, time constant, scan the beginning, the end of the wavelength scanning interval are the same with the results by measuring carbon black), the test samples are measured, and we use the carbon black photo-acoustic signal as the denominator, while the photo-acoustic signal as normalized elements, we can obtain the normalized photo-acoustic spectroscopy. Samples will be displaced and chopper frequency will be changed to obtain photo-acoustic tomography spectroscopy.

\section{Results and Discussion}

\section{Measure of Photo-acoustic Tomography Spectroscopy}

By using the method mentioned above, the photo-acoustic tomography spectrograms of green and yellow leaves from Dracontomelon duperreanum were obtained from different chopping frequency $(22 \mathrm{~Hz}$ and $32 \mathrm{~Hz}$ ) and sample translocation $0.05 \mathrm{~mm}$, respectively, in the time constant of 3s.

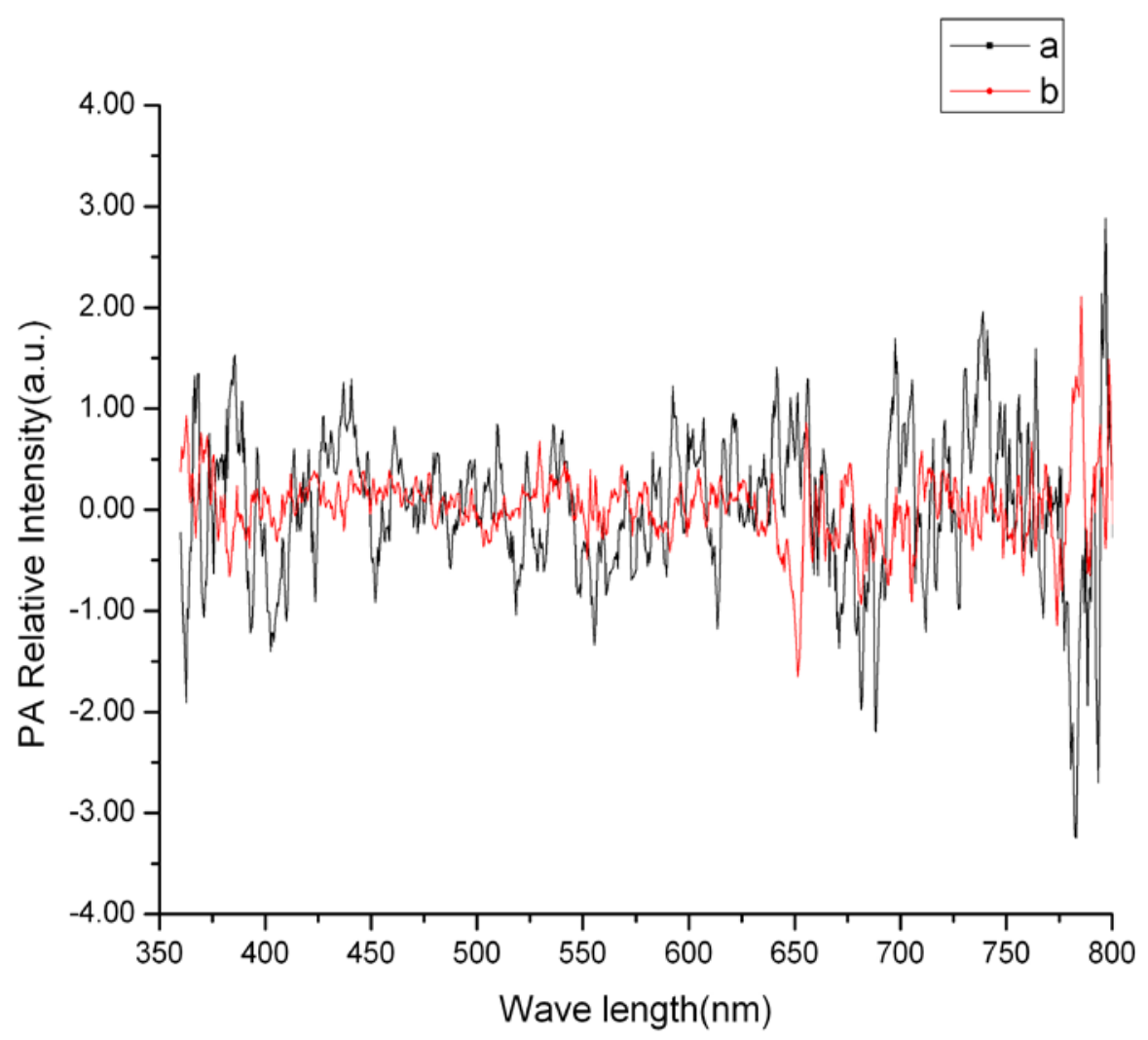

Fig.2 Normalized photo-acoustic tomography spectroscopy of Dracontomelon duperreanum green leaves arranged from top to bottom, immediate measuring of the green leaves front side b, a, were, respectively, in the chopping frequency $22 \mathrm{~Hz}, 32 \mathrm{~Hz}$ and samples were displaced under the conditions of the normalized photo-acoustic tomography spectroscopy. 


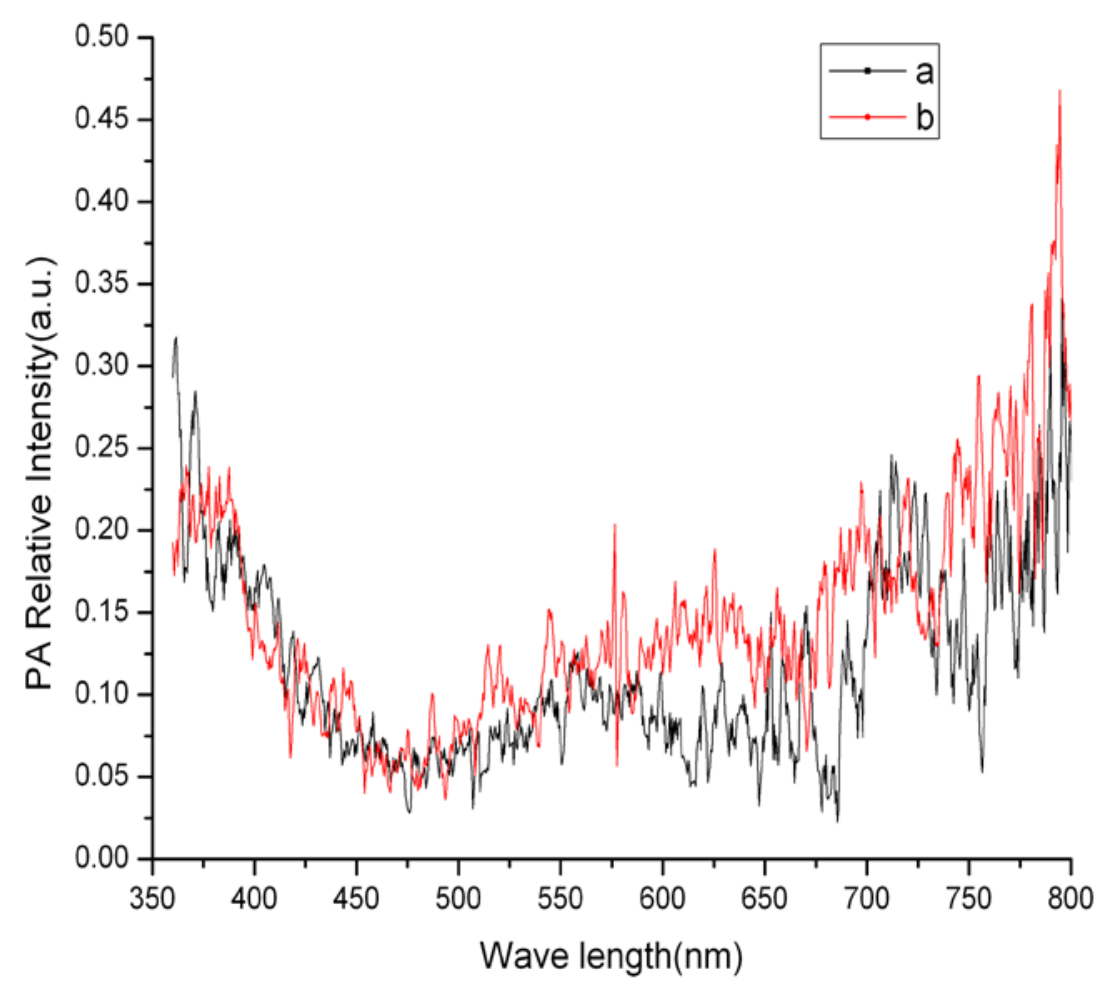

Fig. 3 Normalized photo-acoustic tomography spectroscopy of Dracontomelon duperreanum yellow leaves arranged from top to bottom, immediate measuring of the yellow leaves front side a, b, were, respectively, in the chopping frequency $22 \mathrm{~Hz}, 32 \mathrm{~Hz}$ and samples were displaced under the conditions of the normalized photo-acoustic tomography spectroscopy

According to the results of the Fig. 2 and Fig.3 and discussion can be obtained as follows:

As the spectral absorption coefficient of carbon black is constant(independent of the wavelength), if the spectral absorption coefficient of carbon black unit 1, the absorption peaks of Dracontomelon duperreanum leaves in 350-425nm Soret band,450-550nm carotenoid,600-700nm chlorophyll band with a spectral absorption coefficient of photosynthetic pigment could be measured as shown in Table 1.

Tab. 1 The leaves of Dracontomelon duperreanum spectrum absorption coefficient (in the spectral absorption coefficient of carbon black unit 1)

\begin{tabular}{ccccc}
\hline curva & $\begin{array}{c}\text { Chopping } \\
\text { frequency }\end{array}$ & 350-425nm & 450-550nm & $600-700 \mathrm{~nm}$ \\
\hline Fig.2 $\begin{array}{c}\text { The obverse } \\
\text { of the green } \\
\text { leaves b } \\
\text { The obverse } \\
\text { of the green } \\
\text { leaves a } \\
\text { The obverse } \\
\text { Fig.3 }\end{array}$ & 32Hz & 0.95 & 0.75 & 1.00 \\
$\begin{array}{c}\text { of the yellow } \\
\text { leaves a } \\
\text { The obverse } \\
\text { of the yellow } \\
\text { leaves b }\end{array}$ & 22Hz & $0.32 \mathrm{~Hz}$ & 0.10 & 0.170 \\
\hline
\end{tabular}


As far as we know, the photosynthetic pigment content of yellow leaves are far lower than that of the green ones. According to Fig.2, Fig.3 and Table 1, we found that the more photosynthetic pigment content of Dracontomelon duperreanum leaves is, the bigger the optical absorption coefficient, the higher the photosynthetic efficiency. This is very close to the reported[14]. As we could see from the Fig.2, Fig.3 and Table 1 photosynthetic pigments content of Dracontomelon duperreanum leaves is closely related to optical absorption coefficient, and the technology can be used for photosynthetic research. It makes the measurement of trees’ growth status much easier.

\section{Conclusion}

To measure photo-acoustic tomography spectroscopy of Dracontomelon duperreanum leaves and its optical absorption properties and photosynthesis by a new technique is a kind of good way. The results showed that: The more photosynthetic pigment content of Dracontomelon duperreanum leaves contain, the bigger optical absorption coefficient and the higher photosynthetic efficiency they have. We could find that the photosynthetic pigment content of the green leaves of Dracontomelon duperreanum is higher than that of the yellow ones. As a result, the optical absorption coefficient and the photosynthetic efficiency of the green ones were higher than those of the yellow ones. The non-damage detection study to determine the photosynthesis and status in tree growth is extremely valuable.

\section{Acknowledgement}

This work was supported by NSFC(Under Grant Numbers: 60877068).

\section{References}

[1] Q. F. Xu, J. Yuan, Z. G. Ji and D.L.Que, J.Mater. Sci. and Engineering.17(1999)80.

[2] W. Chen and S. G. Sun, J. Spectroscopy and Spectral Analysis.22(2002)504.

[3] X. Y.Pan and Q.H.Gong, Physics.31(2002)647.

[4] G.Li,M.Zhou,H.J.Wu and L.Lin,J. Spectroscopy and Spectral Analysis.30(2010)2744.

[5] Y. F. Xia, R. S. Liang, Z. L. Tang,Y. Su, J.L. Liu and G. S.Chen,J. Sci. Bulletin.47(2002)1702.

[6] Y.Z.Feng,Z.L.Tang,Y.Q.Xiao,G.S.Chen and J.L.Liu,J.Laser Journal.23(2002)81.

[7] Y.L.Fu,X.B.Zhong,Y.L.Fu and Y.K.Liu, J.Spectroscopy and Spectral Analysis.20(2000)425.

[8] P. Guo, L. E. Liu and Q. Liu, J. Spectroscopy and Spectral Analysis.20 (2000)457.

[9] A. Rosencwaig. and A. Gersho.J.Science.190(1975)556.

[10] A. Rosencwaig. and A. Gersho. J. Appl. Phys. 47(1974)64.

[11] A. Rosencwaig. Opt. Commun.7(1973)305.

[12] D. Cahen, Appl. Phys. Lett. 33(1978)810.

[13] D. Cahen, S. Malkin and E. I. Lerner, FEBS Lett. 91(1978b)339.

[14] A. Rosencwaig, Science,181(1973)657. 